\title{
POSITIVIDADE DO XENODIAGNÓSTICO DE ACORDO COM A FAIXA ETÁRIA, O SEXO E A FORMA CLÍNICA DA DOENÇA DE CHAGAS
}

\author{
Ionizete Garcia da Silva*, Heloisa Helena Garcia da Silva**, Alejandro Luquetti \\ Ostermayer ", Joffre Marcondes de Rezende
}

\section{RESUMO}

O xenodiagnóstico foi aplicado em 76 pacientes chagásicos na fase crônica, dos quais eram conhecidos os exames clínico e sorológicos, e nenhum dos pacientes tinha sido submetido a tratamento específico. Em cada xenodiagnóstico utilizou-se o mínimo de 36 triatomíneos distribuídos equitativamente entre as quatro espécies. A positividade do xenodiagnóstico foi de $55,3 \%$ e mostrou-se independente da faixa etária, do sexo e da forma clínica da doença.

UNITERMOS: Xenodiagnóstico. Doença de Chagas. Trypanosoma cruzi. Forma clínica. Triatomíneos.

\section{INTRODUÇÃO}

O xenodiagnóstico é um exame que consiste na deteç̧ão do Trypanosoma cruzi através da pesquisa do parasito nas fezes e/ou urina dos triatomíneos. Tem sido utilizado na comprovação parasitológica da doença de Chagas na fase crônica e, também, como método de seleção de pacientes e/ou na avaliação de tratamento específico ${ }^{1,3,4,6,11}$. Este estudo teve a finalidade de verificar se a positividade do xenodiagnóstico estava associada à forma clínica, ao sexo e à idade dos pacientes.

\footnotetext{
- Prof.do Depto. de Parasitologia - IPTSP/UFG.

"Farmacêutica do Depto. de Parasitologia -IPTSP/UFG

"."Prof. da Faculdade de Medicina/UFG.

Apoio financeiro - SENESU/PRPPG/FUNAPE

Recebido para publicação em 18/09/95
} 
SILVA, I.G.; SILVA, H.H.G.; LUQUETTI, A.O.; \& REZENDE, J.M.Positividade do xenodiagnóstico de acordo com a faixa etária, o sexo e a forma clínica da Doença de Chagas. Rev. Pat. Trop.24 ( 2 ): 193-197, jul/dez. 1995.

\section{MATERIAL E MÉTODOS}

Espécies utilizadas - Utilizaram-se ninfas de $1^{\circ}$ estádio de Dipetalogaster maximus, cerca de 15 dias após a eclosão das mesmas ${ }^{8}$, e ninfas de $4^{\circ}$ e $5^{\circ}$ estádios das espécies: Panstrongylus megistus, Rhodnius nasutus, $R$. neglectus, $R$. prolixus, $R$. robustus, Triatoma infestans. T. rubrovaria e T. vitticeps. Todas estas espécies foram testadas e se apresentaram como replicadoras de $T$. cruzi ${ }^{10,11,12}$. As ninfas foram criadas e mantidas em câmara biológica. Logo após a ecdise, as ninfas eram colocadas em frascos apropriados ${ }^{7}$ ao xenodiagnóstico, permanecendo nestes, em jejum, por 25 e 30 dias, para o $4^{\circ}$ e $5^{\circ}$ estádios, respectivamente. $\mathrm{O}$ xenodiagnóstico foi realizado de acordo com técnica já estabelecida ${ }^{9}$. Colocaram-se 12 frascos por paciente e procurou-se distribuí-los com eqüidade por espécie, segundo a disponibilidade.

Xenodiagnóstico - Foi aplicado em 76 pacientes chagásicos crônicos do Hospital das Clínicas da Universidade Federal de Goiás, dos quais eram conhecidos previamente os exames clínico e sorológicos, e nenhum dos pacientes havia sido submetido a tratamento especifico.

Leituras - Foram realizadas leituras aos 30,60 e 90 dias, após a aplicação do xenodiagnóstico, utilizando-se o método das dejeções espontâneas ${ }^{9}$.

Análise estatística - O teste do Qui-quadrado foi usado para comparação dos valores obtidos da positividade do xenodiagnóstico, em pacientes chagásicos crônicos, de acordo com a faixa etária, com o sexo e com a forma clínica da doença.

\section{RESULTADOS E DISCUSSÃO}

Os resultados encontram-se nas Tabelas 1 e 2 . Constatou-se que a positividade do xenodiagnóstico, nos 76 chagásicos crônicos, foi independente da faixa etária, do sexo e da forma clínica da doença, pelo teste do Qui-quadrado, ao nível de $5 \%$ (Tabelas 1,2 ).

No sexo masculino, a positividade foi maior na faixa etária de 10 a 20 anos, e no feminino, nas faixas etárias de 31 a 40 e de 61 a 72 anos. Isto é concordante com dados da literatura. A positividade geral entre todas as faixas etárias foi de $55,3 \%$.
SILVA, I.G.; SILVA, H.H.G.; LUQUETTI, A.O.; \& REZENDE, J.M.Positividade do xenodiagnóstico de acordo com a faixa etária, o sexo e a forma clínica da Doença de Chagas. Rev. Pat. Trop.24 ( 2 ): 193-197, jul/dez. 1995

Tabela 1. Positividade do xenodiagnóstico em 76 pacientes chagásicos crônicos, com sorologia reativa, de acordo com a faixa etária, sexo e forma clínica da doença.

\begin{tabular}{|c|c|c|c|c|c|c|c|c|}
\hline \multirow[t]{2}{*}{$\begin{array}{c}\text { FAIXA } \\
\text { ETÁRIA }\end{array}$} & \multicolumn{2}{|c|}{ MASCULINO } & \multicolumn{2}{|c|}{ FEMININO } & \multicolumn{2}{|c|}{ FORMA CLÍNICA } & \multicolumn{2}{|c|}{ TOTAL } \\
\hline & NP/NE & $\%$ & $\mathrm{NP} / \mathrm{NE}$ & $\%$ & MEGA & CARD-MEGA & $\overline{\mathrm{NP} / \mathrm{NE}}$ & $\%$ \\
\hline $10-20$ & $6 / 6$ & 100,0 & $0 / 1$ & 0,0 & 5 & 2 & $6 / 7$ & 85,7 \\
\hline $21-30$ & $3 / 5$ & 60,0 & $2 / 4$ & 50,0 & 6 & 3 & $5 / 9$ & 55,6 \\
\hline $31-40$ & $6 / 14$ & 42,9 & $5 / 6$ & 83,3 & 16 & 4 & $11 / 20$ & 55,0 \\
\hline $41-50$ & $6 / 13$ & 46,2 & $1 / 5$ & 20,0 & 13 & 5 & $7 / 18$ & 38,9 \\
\hline $51-60$ & $4 / 7$ & 57,1 & $4 / 6$ & 66,7 & 9 & 4 & $8 / 13$ & 61,5 \\
\hline $61-72$ & $1 / 4$ & 25,0 & $4 / 5$ & 80,0 & 7 & 2 & $5 / 9$ & 55,6 \\
\hline & $26 / 49$ & 53,1 & $16 / 27$ & 59,3 & 56 & 20 & $42 / 76$ & 55,3 \\
\hline
\end{tabular}

$\mathrm{NP}=$ Número de pacientes positivos.

$\mathrm{NE}=$ Número de pacientes examinados.

MEGA $=$ Megaesôfago e/ou megacólon.

$\mathrm{CARD}=$ Cardiopatia

O xenodiagnóstico quando aplicado em população não selecionada, clínica e sorologicamente, apresenta baixa positividade ${ }^{2}$. Quando selecionada, a positividade é variável, geralmente entre 30 a $40 \%$, de acordo com a metodologia de aplicação de xenodiagnóstico $1,3,4,5,6,7,8$

Tabela 2. Positividade do xenodiagnóstico em 76 pacientes chagásicos crônicos, de acordo com o número de triatomíneos infectados com o Trypanosoma cruzi.

\begin{tabular}{lccrc}
\hline \multirow{2}{*}{ PARASITEMIA } & $N^{\circ}$ DE PACIENTES & N $^{0}$ & $\begin{array}{r}\text { DE } \\
\text { INFECTADOS/EXAMINADOS }\end{array}$ & $\%$ \\
\hline ALTA & 6 & $142 / 203$ & 70,0 \\
MÉDIA & 23 & $180 / 1.313$ & 13,7 \\
BAIXA & 47 & $20 / 2.218$ & 1,0 \\
\hline
\end{tabular}

Pela percentagem do número de triatomíneos positivos em relação aos examinados, estimou-se a parasitemia dos pacientes, em três níveis: alta, média e baixa. A primeira caracterizou-se pela facilidade de se detectar o tripanosoma e 
SILVA, I.G.; SILVA, H.H.G.; LUQUETTI, A.O.; \& REZENDE, J.M.Positividade do xenodiagnóstico de acordo com a faixa etária, o sexo e a forma clínica da Doença de Chagas. Rev. Pat. Trop.24 ( 2 ): 193-197, jul/dez. 1995.

corresponde aos pacientes com mais de $50 \%$ de triatomíneos infectados pelo $T$. cruzi; a segunda apresentou um grau de dificuldade intermediário, corresponde a uma frequêencia de infecção entre 5 e $50 \%$; a terceira foi o nível de mais difícil detecção do tripanosoma, com menos de $5 \%$.

A distribuição do número de pacientes, de acordo com a parasitemia alta, média e baixa, foi, respectivamente, de $7,9 \%, 30 \%$ e $61,8 \%$. Esta distribuição foi semelhante à de um trabalho ${ }^{1}$ realizado em Mambaí/GO, no qual os autores obtiveram $9,2 \%, 24,1 \%$ e $66,7 \%$, de um total de 303 pacientes chagásicos crônicos estudados. A maioria dos pacientes apresentou baixa parasitemia, o que deve ter sido um fator negativo e determinante na sensibilidade do xenodiagnóstico. Este fato pode sinalizar um ponto, em torno do qual deve-se investigar, na tentativa de aprimorar o método, incorporando mecanismos que melhorem sua sensibilidade.

\section{SUMMARY}

Relation between parasitemia detected by xenodiagnosis, age and sex in patients at the chronic phase of Chagas' disease

Seventy-six chronic phase chagasic patients, with known clinical and serology examinations, were submited to xenodiagnosis. None of the patients have had specific treatment before. Thirty-six bugs, equally distributed in 4 species, were used in each xenodiagnosis. The xenodiagnosis positivity was of $55,3 \%$, and showed no correlation between age, sex, and clinical form of the disease.

KEYWORDS: Xenodiagnosis. Chagas' disease. Trypanosoma cruzi. Triatomines.

\section{REFERÊNCIAS BIBLIOGRÁFICAS}

01.CASTRO, C.N.; ALVES, M.T. \& MACEDO, V.O. Importância da repetição do xenodiagnóstico para avaliação da parasitemia na fase crônica da doença de Chagas. Rev.Soc.Bras.Med. Trop., 16:98-103, 1983.

02.DIAS, E. Técnica do xenodiagnóstico na moléstia de Chagas. Mem.Inst.Oswaldo Cruz, 35:335-344, 1940.

03.FREITAS, J.L.P. de. O diagnóstico de laboratório da moléstia de Chagas. Rev.Clín., S.Paulo, 28:11-20, 1952.
SILVA, I.G.; SILVA, H.H.G.; LUQUETTI, A.O.; \& REZENDE, J.M.Positividade do xenodiagnóstico de acordo com a faixa etária, o sexo e a forma clínica da Doença de Chagas. Rev. Pat. Trop.24 ( 2 ): 193-197, jul/dez. 1995.

04.MARSDEN, P.D,; BARRETO, A.C.; CUBA, C.C.; GAMA, M.B. \& AKERS, J. Improvements in routine xenodiagnosis with first instar Dipetalogaster maximus (Uhler, 1894) (Triatominae). Am.J.Trop.Med.Hyg., 28:649-652, 1979.

05.SCHENONE, H.; ALFARO, E.; ROJAS, A. Bases y rendimiento del xenodiagnóstico en la infección chagásica humana. Bol. Chil.Parasitol., 29:24-26, 1974.

06.SCHENONE, H.; ROJO, M.; ROJAS, A.; CONCHA, L. Positividad diurna y nocturna del xenodiagnóstico en un paciente con infección chagásica crónica de parasitemia permanente. Bol.Chil. Parasitol., 32:63-66, 1977.

07. SILVA, I.G.da Influência da temperatura na biologia de triatomíneos.I.Triatoma rubrovaria (Blanchard, 1843) (Hemiptera, Reduviidae).Rev.Goiana Med., 31:1-37, 1985.

08. SILVA, I.G.da Influência da temperatura na biologia de triatomíneos. XIII. Dipetalogaster maximus Uhler, 1894 (Hemiptera, Reduviidae).Anais Soc.Ent.Bras., 19:111-119, 1990a.

09. SILVA, I.G. da Nova técnica para leitura do xenodiagnóstico. Rev.Goiana Med., 36:35-40, $1990 \mathrm{~b}$.

10. SILVA, I.G. da \& SILVA, H.H.G. da Suscetibilidade de 11 espécies de triatomíneos (Hemiptera, Reduviidae) à cepa $Y$ de Trypanosoma cruzi (Kinetoplastida, Trypanosomatidae). Rev.Bras.Ent., 37:459-463, 1993.

11. SILVA, I.G. da; LUQUETTI, A.O \& SILVA, H.H.G. da. Importância do método de obtenção das dejeções espontâneas dos triatomíneos na avaliação da suscetibilidade triatomínica para o Trypanosoma cruzi. Rev.Soc.Bras.Med. Trop., 26: 19-24, 1993.

12. SILVA, I.G. da; NAKANO, H.; SILVA, H.H.G. da; NAKANO, R. Estudo da suscetibilidade de diferentes espécies de triatomíneos (Hemiptera, Reduviidae) ao Trypanosoma cruzi (Kinetoplastida, Trypanosomatidae). Rev. Soc. Ent. Bras., 23:495-511, 1994. 